\title{
Temporal-based approach to solve item decay problem in recommendation system
}

\begin{abstract}
The rating matrix of a recommendation system contains a high percentage of data sparsity which lowers the prediction accuracy of the collaborative filtering technique $(\mathrm{CF})$. Recently, the temporal based factorization approaches have been used to solve the sparsity problem, but these approaches have a weakness in terms of learning the popularity decay of items during the long-term which lowers the prediction accuracy of the $\mathrm{CF}$ technique. The LongTemporalMF approach has been proposed to solve these problems. The x-means algorithm and the bacterial foraging optimization algorithm have been integrated within the LongTemporalMF approach to generate and optimize the genres weights which are integrated with the factorization features and the long-term preferences in terms of personality. The experimental results show that the LongTemporalMF approach has the accurate prediction performance compared to the benchmark approaches.
\end{abstract}

Keyword: Bacterial foraging; Clustering; Collaborative filtering; Data sparsity; Matrix factorization; Recommendation system 